\title{
Correlation of clinical features and genetic profiles of stromal interaction molecule 1 (STIM1) in colorectal cancers
}

\author{
Henry Sung-Ching Wong ${ }^{1,2}$, Wei-Chiao Chang ${ }^{1,2,3,4,5}$ \\ ${ }^{1}$ Master Program for Clinical Pharmacogenomics and Pharmacoproteomics, School of Pharmacy, Taipei Medical University, \\ Taipei, Taiwan \\ ${ }^{2}$ Department of Clinical Pharmacy, School of Pharmacy, Taipei Medical University, Taipei, Taiwan \\ ${ }^{3}$ Department of Pharmacy, Taipei Medical University Wan Fang Hospital, Taipei, Taiwan \\ ${ }^{4}$ Center for Biomarkers and Biotech Drugs, Kaohsiung Medical University, Kaohsiung, Taiwan \\ ${ }^{5}$ Department of Pharmacy, Taipei Medical University Wan Fang Hospital, Taipei, Taiwan \\ Correspondence to: \\ Wei-Chiao Chang, e-mail: wcc@tmu.edu.tw \\ Keywords: colorectal cancer, stored-operated calcium entry pathway, stromal interaction molecule 1, bioinformatics, data \\ mining \\ Received: July 21, $2015 \quad$ Accepted: October 22, $2015 \quad$ Published: November 03, 2015
}

\section{ABSTRACT}

STIM1 overexpression has been observed in a portion of colorectal cancer (CRC) patients and associated with cancer cell invasion and migration. To characterize the distinctive expression profiles associated with stromal interaction molecule 1 (STIM1) overexpression/low-expression between CRC subtypes, and further assess the divergence transcription regulation impact of STIM1 between colon (COADs) and rectum (READs) adenocarcinomas in order to depict the role of SOCE pathway in CRCs, we have conducted a comprehensive phenome-transcriptome-interactome analysis to clarify underlying molecular differences of COADs/READs contributed by STIM1. Results demonstrated that a number of novel STIM1-associated signatures have been identified in COADs but not READs. Specifically, the presence of STIM1 overexpression in COADs, which represented a disturbance of the SOCE pathway, was associated with cell migration and cell motility properties. We identified 11 prognostic mRNA/ miRNA predictors associated with the overall survival of COAD patients, suggesting the correlation of STIM1-associated features to clinicopathological outcomes. These findings enhance our understanding on differences between CRC subtypes in panoramic view, and suggested STIM1 as a promising therapeutic biomarker in COADs.

\section{INTRODUCTION}

Colorectal cancer (CRC) is the fourth most frequent human malignancy in men and is the third most frequent in women worldwide, as well as the second most common cause of cancer-related morbidity and mortality [1]. CRC transformation from the normal colonic mucosa arises through a progressive accumulation of genetic and epigenetic changes. In CRC, colon adenocarcinomas (COADs) and rectal adenocarcinomas (READs) are largely anatomically diverse CRC classifications. However, the correlation between molecular profiles and anatomic-classified COADs/READs is inconspicuous. In addition, epidemiological evidence revealed that colon and rectal carcinomas differ in terms of their prognoses, with colon cancer showing a greater aggressiveness and poorer clinical outcomes than rectal cancer.

The invasion-metastasis cascade involves sequential steps of local invasion, intravasation, transition, extravasation, and colonization which can be used to describe the distinctive nature of carcinomas [2]. Carcinomas at the invasive front undergo "the epithelial-mesenchymal transition (EMT)" which enables progression to occur, a process which involves multiple signaling pathway changes in CRC including transforming growth factor (TGF)- $\beta$ signaling, WNT signaling, and so on. However, recent microarray profiling techniques have identified different expression features between colon and rectal carcinomas [3], and demonstrated substantial biological heterogeneity between colon and rectal 
carcinomas. Despite the identified pathway differences between colon and rectal carcinomas, the molecular or pathway root causes of cancer aggressiveness differ in colon/rectal carcinomas, and differences that underlie the prognosis potential remain obscure.

Recent studies have illustrated that $\mathrm{Ca}^{2+}$ signaling is increasingly implicated in CRC invasion and metastasis. The predominant $\mathrm{Ca}^{2+}$ signaling mechanism in most tumor cells is store-operated $\mathrm{Ca}^{2+}$ entry (SOCE), and SOCE-mediated $\mathrm{Ca}^{2+}$ oscillation is critical for focalized proteolysis, which is exploited by cancers to accelerate invasion and metastasis. The endoplasmic reticular (ER) $\mathrm{Ca}^{2+}$ sensor, stromal interaction molecule 1 (STIM1), which regulates the $\mathrm{Ca}^{2+}$ entry process in response to external stimuli, is thought to play a central role in coordinating $\mathrm{Ca}^{2+}$ signaling. It is known that STIM1-mediated $\mathrm{Ca}^{2+}$ oscillation controls invadopodium formation and focal adhesion turnover, and ultimately orchestrates tumor cell invasion and migration [4, 5]. In our previous study on CRC, STIM1 overexpression increased CRC aggressiveness, COX-2 gene activation and promoted tumor progression $[6,14]$. In addition, blockage of the SOCE alleviated the aggressiveness of tumor cells. Those results suggested the importance of STIM1 overexpression in the tumor invasion-metastasis cascade, and therefore STIM1 may be developed as a potential therapeutic target of cancer treatment. Nevertheless, impacts of $\mathrm{Ca}^{2+}$ signaling and SOCE pathway aberrations on different CRC subtypes, i.e., colon and rectal carcinomas, remain exclusive. To address these issues, we conducted an integrated analysis focused on the transcriptome and interactome in colon and rectal carcinomas with distinctive invasiveness and aggressiveness features.

The Cancer Genome Atlas (TCGA) project focusing on CRC was carried out at 2012 [7], and data are available through its data portal. Using biological computational techniques, we comprehensively analyzed all available transcriptomic profiles (including messenger (m)RNA and micro (mi)RNA data) to gain a panoramic view of expression patterns between colon and rectal cancers. Furthermore, correlation analyses between tumor aggressiveness behaviors and expression patterns were carried out to point out the fundamental molecular contributions to the clinical tumor invasion status.

\section{RESULTS}

\section{Correlation between patients' clinical profile and STIM1 expression values in CRC}

CRC patients with available STIM1 expression data were separated into COAD $(n=154)$ and READ $(n=68)$ groups (Figure 1A). In each CRC subtype, correlation analyses between STIM1 expression values and clinical profiles were conducted. First, we assessed the STIMI expression value between COAD and READ patients, and the expression value of STIM1 was significantly higher in COAD (mean $\pm \mathrm{SD}=0.144 \pm 1.065$ ) than READ (mean $\pm \mathrm{SD}=-0.202 \pm 0.906$ ) patients (exact Wilcoxon Mann-Whitney rank sum $p=0.0066$, Figure $1 \mathrm{~B})$. In the dichotomized STIM1 group, 27 of 154 (17.5\%) were STIM1 overexpression and 20 of $154(13.0 \%)$ were STIMI low-expression in COADs. In READs, only 5 of $68(7.4 \%)$ were STIM1 overexpression and 17 of $68(25.0 \%)$ were STIM1 low-expression.

To examine the clinicopathological role of STIM1 in CRC, summaries (Table 1) and associations (Table 2) between STIM1 z-scores and several clinical features were separately examined in COADs and READs. As shown in Table 2, a correlation between STIM1 and lymphatic invasion was observed ( $p=0.0253$, odds ratio (OR) $=1.4515,95 \%$ confidence interval $(\mathrm{CI})=1.06 \sim 2.03)$ in COADs. STIM1 also showed a modest borderline significant correlation with the vascular invasion status $(\mathrm{OR}=1.4474,95 \% \mathrm{CI}=0.99 \sim 2.15)$, but a significant threshold was not reached $(p=0.0593)$. However, there were no statistically significant associations of STIMI with the disease stage, tumor stage, lymph node spread status, or distant metastasis status. In contrast, READs did not reach a significant threshold in either lymphatic invasion or vascular invasion, which implied a possible significance of STIM's role in COADs but not ROADs, further suggesting hyperactivation of the SOCE pathway in COAD patients.

\section{STIM1-associated genes differentially expressed in COADs but not READs}

COAD and READ patients were further categorized into a STIM1 overexpression group and STIM1 lowexpression group. Patients with STIM1 z-scores of $>+1$ were categorized into the STIM1 overexpression group (STIM1+), and patients with STIM1 z-scores of <-1 were categorized into the STIM1 low-expression group (STIM1-). According to these criteria, 47 COAD patients (including 27 STIM1 overexpression and 20 STIM1 low-expression) and 22 READ patients (including 5 STIM1 overexpression and 17 STIM1 low-expression) were subjected to a microarray analysis. DEGs were identified in 69 CRC patients' microarray data. Moderated t-statistics were calculated to identify DEGs in each CRC subtype. In COAD patients, 306 upregulated DEGs and 139 downregulated DEGs were identified and fulfilled the FDR-adjusted $p$ value criteria of $<0.1$ among the STIM1 overexpression group and STIM1 low-expression group (Supplementary Table S1A). Intriguingly, neither significant upregulated nor downregulated DEGs were detected in READ patients. This implied that STIMI could be an important marker to distinguish COAD patients from READ patients.

We further identified co-expression patterns between COAD and READ patients. Gene lists between COAD and READ patients were compared using a $\log 2$ multiple of 
A

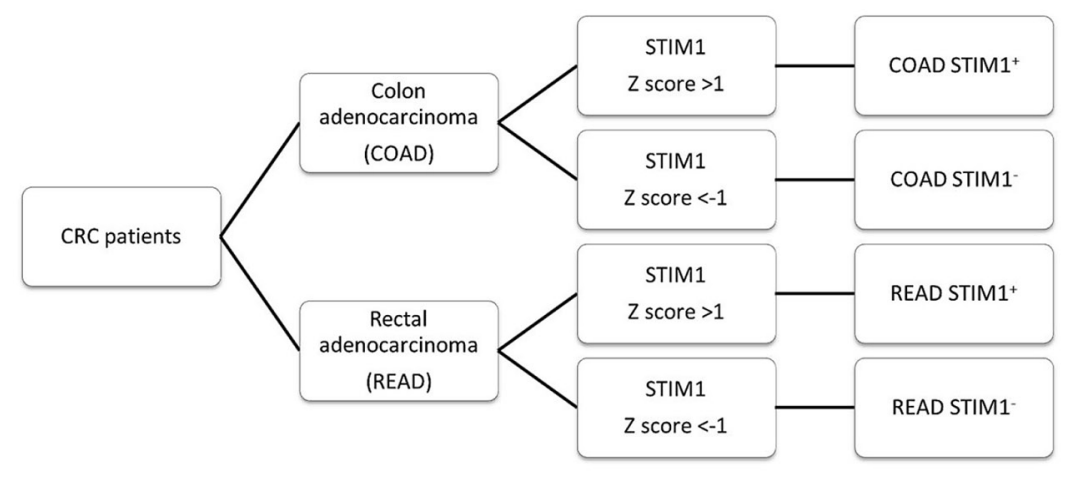

B

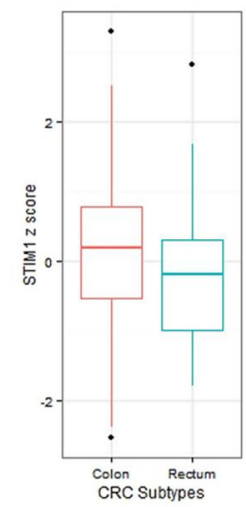

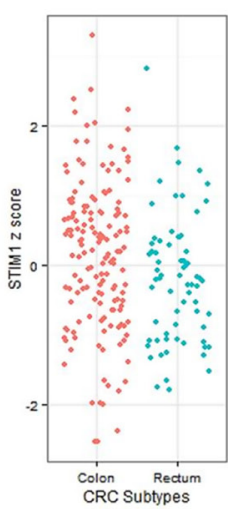

Figure 1: Patient categorization for STIM1-mediated $\mathrm{Ca}^{2+}$ signaling analysis based on colorectal cancer (CRC) subtypes and the STIMI expression status. A. Patient selection criteria used for the following analysis (microarray, mRNA sequencing, and miRNA sequencing data). CRC patients were anatomically categorized into colon adenocarcinomas (COADs) and rectal adenocarcinomas (READs). These patients were further stratified into STIM1+ and STIM1- groups based on the presence or absence of STIM1 overexpression. Patients with a STIM1 z score of $>+1$ or $<-1$ were selected to undergo differentially expressed gene identification. B. A whisker boxplot and jitter plot summarizing differences in normalized STIM1 expression levels ( $\mathrm{Z}$ scores) among COADs and READs (Wilcoxon Mann-Whitney rank sum $p=0.0066$ ).

change cutoff of \pm 1.5 (Figure 2A). Numbers of genes that showed a homodirectional pattern and opposite changes, and were uniquely differentially expressed in COADs or READs were quantified (Figure 2B). In 2033 selected genes based on $\log 2$ multiples of change, $614(30.2 \%)$ and 724 $(35.6 \%)$ were respectively downregulated and upregulated in only READs. In only COADs, 118 (5.8\%) and 324 $(15.9 \%)$ were respectively downregulated and upregulated. There were $79(3.9 \%)$ and $143(7.0 \%)$ genes which respectively showed downregulation and upregulation in both CRC subtypes (homodirectional); while 9 (0.44\%) and $22(1.1 \%)$ showed opposite directions in expression patterns across CRC subtypes (Supplementary Table S2). The Spearman rank correlation test revealed a correlation coefficient of $0.39(p<0.01)$, indicating a low similarity of expression profiles between COADs and READs. Unsupervised agglomerative hierarchical clustering analyses were conducted to clarify the aggregative effect of STIM1 expression patterns based on microarray profiles. The top 100 most significantly DEGs identified by the FDR-adjusted $p$ value in COADs were clustered, and results are shown for each CRC subtype. As READs showed no significantly up- or downregulated DEGs, no genes were selected for clustering. In COADs, a clear clustering pattern was observed, and a gathering configuration on STIM1 overexpression patients and STIM1 low-expression patients was clearly distinguishable (Figure 2C), revealing a highly similar within-group expression pattern in COAD patients. However, a recognizable pattern was no longer visible in READs when we used the same top 100 most significant DEGs for READ clustering (Figure 2D). This result indicated that remarkable latent molecular signatures could be used to distinguish COADs and READs.

Following the clustering analysis, a two-step NMF was carried out against the top 100 DEGs. First, expression values were transformed into non-negative values (Supplementary Figure S1A). Then, Brunet et al.'s algorithm [8] was performed on the factorization rank between 2 and 6 in 200 runs to identify the optimal number of clusters. With the aid of visualization by a consensus matrix, the strongest consensus signature was observed with a factorization rank of 2 (Supplementary Figure S1B). The result indicated that the NMF algorithm could attain good clustering stability in a factorization rank of 2 that consistently clustered patients in each run based on STIM1 expression features. This was consistent with the hierarchical clustering result, that COAD patients were well-categorized into STIM1 high-expression and STIM1 low-expression groups based on two metagenes (Supplementary Figure S1C).

A series of analyses revealed that (i) the underlying molecular schemes of COADs and READs differed; (ii) STIM1 could be a biomarker for COADs but not READs, and (iii) there were differences in fundamental biological pathways between COADs and READs.

\section{Prospecting underlying biological features in COADs and READs}

For microarray expression profiles of COAD and READ patients, all signatures were included in the GSEA to detect coordinated changes in the same BP terms. Outcome enrichment scores were further normalized for comparisons (Supplementary Tables S3, S4). After filtering, differences in normalized enrichment scores were compared between COADs and READs (Supplementary Figure S2). Note that only the BP terms with a value of $\mathrm{NES}_{\mathrm{COAD}}-\mathrm{NES}_{\mathrm{READ}}$ of $>0$ (which means a positive value) were of interest. Therefore, BP terms identified to be 
Table 1: Clinicopathological features of 222 colorectal cancer patients

\begin{tabular}{|c|c|c|c|c|}
\hline Characteristics & Total cases $N$ & $C O A D s N(\%)$ & $R E A D s N(\%)$ & P-value \\
\hline \multicolumn{5}{|l|}{ Gender } \\
\hline Male & 116 & $79(68.10)$ & $37(31.90)$ & $0.771^{\mathrm{a}}$ \\
\hline Female & 106 & $75(70.75)$ & $31(29.25)$ & \\
\hline \multicolumn{5}{|l|}{ Age (years) } \\
\hline Mean $\pm S D$ & 222 & $70.66 \pm 11.65$ & $66.63 \pm 10.76$ & $0.004^{\mathrm{b}}$ \\
\hline Range & & $36-90$ & $35-89$ & \\
\hline \multicolumn{5}{|c|}{ Depth of tumor invasion } \\
\hline $\mathrm{T} 1+\mathrm{T} 2$ & 55 & $35(63.64)$ & $20(36.36)$ & $0.396^{\mathrm{a}}$ \\
\hline $\mathrm{T} 3+\mathrm{T} 4$ & 149 & $105(70.47)$ & $44(29.53)$ & \\
\hline \multicolumn{5}{|c|}{ Lymph node metastasis } \\
\hline No & 136 & $94(69.12)$ & $42(30.88)$ & $0.839^{\mathrm{a}}$ \\
\hline N1 & 42 & $28(66.67)$ & $14(33.33)$ & \\
\hline $\mathrm{N} 2$ & 44 & $32(72.73)$ & $12(27.27)$ & \\
\hline \multicolumn{5}{|c|}{ Distant Metastasis } \\
\hline No & 185 & $129(69.73)$ & $56(30.27)$ & $0.552^{\mathrm{a}}$ \\
\hline Yes & 34 & $22(64.71)$ & $12(35.29)$ & \\
\hline \multicolumn{5}{|l|}{ Stage (UICC) } \\
\hline $\mathrm{I}+\mathrm{II}(\%)$ & 131 & $91(69.47)$ & $40(30.53)$ & $1.000^{\mathrm{a}}$ \\
\hline III + IV (\%) & 88 & $61(69.32)$ & $27(30.68)$ & \\
\hline \multicolumn{5}{|c|}{ Vascular invasion } \\
\hline No & 153 & $106(69.28)$ & $47(30.72)$ & $0.730^{\mathrm{a}}$ \\
\hline Yes & 51 & $34(66.67)$ & $17(33.33)$ & \\
\hline \multicolumn{5}{|c|}{ Lymphatic invasion } \\
\hline No & 101 & $71(70.30)$ & $30(29.70)$ & $0.882^{\mathrm{a}}$ \\
\hline Yes & 113 & $78(69.03)$ & 35 (30.97) & \\
\hline
\end{tabular}

Abbrevations: SD, standard deviation; COAD, colon adenocarcinoma; READ, rectal adenocarcinoma; N, number. ${ }^{a} P$-values were calculated by Fisher's exact test.

${ }^{\mathrm{b}} P$-value was calculated by Wilcoxon test.

significantly enriched in COADs compared to READs were "immune system process" (GO:0002376), "regulation of cell migration" (GO:0030334) and "regulation of cell motility" (GO:2000145) (Supplementary Figure S3). In particular, the BP terms of "regulation of cell migration" and "regulation of cell motility" could work in concert with clinical features of STIM1, which is associated with lymphatic invasion in COAD patients. Specifically, the enriched BP terms provided support for the involvement of STIM1 and STIM1-related molecular signatures in tumor invasion progress. This result also indicated a similar enriched BP (which represented common CRC or cancer features) but different subtype-related molecular signatures (which represented subtype-enriched BPs) in CRC patients.

\section{Validation of distinctive STIM1 roles in CRC subtypes by RNA-sequencing analysis and a pathway topology-based approach}

RNA-sequence RPKM values were analyzed to elucidate the subtype-specific effect of STIM1 in CRC. In total, 56 COAD patients (including 26 STIM1 overexpression and 30 STIM1 low-expression) and 21 READ patients (including 11 STIM1 overexpression and 10 STIM1 low-expression) were investigated. To harmonize the RPKM value to downstream differential expression identification, we rounded RPKM values below 0.1 to prevent the deviation caused by lowcoverage genes and then used a $\log 2$ transformation to 
Table 2: Logistic regression analysis between STIM1 expression value to clinical features in colon adenocarcinoma (COAD) and rectum adenocarcinoma (READ) patients

\begin{tabular}{|c|c|c|c|c|c|c|c|c|}
\hline & \multicolumn{4}{|c|}{ COAD $^{a}$} & \multicolumn{4}{|c|}{ READ $^{a}$} \\
\hline & No. of pts & $\mathbf{O R}^{\mathbf{b}}$ & $95 \% \mathrm{CI}^{\mathrm{c}}$ & $P$-value & No. of pts & $\mathbf{O R}^{\mathbf{b}}$ & $95 \% \mathrm{CI}^{\mathrm{c}}$ & $P$-value \\
\hline Stage $e^{d}$ & $\begin{array}{l}0: 91 \\
1: 61\end{array}$ & 0.9450 & $0.69-1.28$ & 0.7176 & $\begin{array}{l}0: 40 \\
1: 27\end{array}$ & 0.9642 & $0.55-1.67$ & 0.8961 \\
\hline $\begin{array}{l}\text { Depth } \\
\text { of tumor } \\
\text { invasion }\end{array}$ & $\begin{array}{c}0: 49 \\
1: 105\end{array}$ & 1.0534 & $0.76-1.45$ & 0.7499 & $\begin{array}{l}0: 24 \\
1: 44\end{array}$ & 1.4404 & $0.81-2.72$ & 0.2380 \\
\hline $\begin{array}{l}\text { Lymph node } \\
\text { metastasis }^{f}\end{array}$ & $\begin{array}{l}\text { N0: } 94 \\
\text { N1: } 28 \\
\text { N2: } 32\end{array}$ & $\begin{array}{c}\mathrm{N} 1: \\
0.8081 \\
\mathrm{~N} 2: \\
1.2040\end{array}$ & $\begin{array}{c}\mathrm{N} 1: \\
0.54-1.21 \\
\mathrm{~N} 2: \\
0.82-1.77\end{array}$ & $\begin{array}{c}\text { N1: } \\
0.2982 \\
\text { N2: } \\
0.3438\end{array}$ & $\begin{array}{l}\text { N0: } 42 \\
\text { N1: } 14 \\
\text { N2: } 12\end{array}$ & $\begin{array}{l}\text { N1: } 1.1869 \\
\text { N2: } 0.6230\end{array}$ & $\begin{array}{c}\mathrm{N} 1: \\
0.62-2.26 \\
\mathrm{~N} 2: \\
0.28-1.39\end{array}$ & $\begin{array}{c}\text { N1: } \\
0.6012 \\
\text { N2: } \\
0.2468\end{array}$ \\
\hline $\begin{array}{l}\text { Distant } \\
\text { Metastasisg }\end{array}$ & $\begin{array}{c}\text { M0: } 129 \\
\text { M1: } 22\end{array}$ & 1.3144 & $0.86-2.04$ & 0.2138 & $\begin{array}{l}\text { M0: } 56 \\
\text { M1: } 12\end{array}$ & 0.9040 & $0.42-1.81$ & 0.7834 \\
\hline $\begin{array}{l}\text { Vascular } \\
\text { Invasion }\end{array}$ & $\begin{array}{l}\text { No: } 106 \\
\text { Yes: } 34\end{array}$ & 1.4474 & $0.99-2.15$ & 0.0593 & $\begin{array}{l}\text { No: } 47 \\
\text { Yes: } 17\end{array}$ & 0.7759 & $0.38-1.53$ & 0.4738 \\
\hline $\begin{array}{l}\text { Lymphatic } \\
\text { Invasion }\end{array}$ & $\begin{array}{l}\text { No: } 72 \\
\text { Yes: } 77\end{array}$ & 1.4515 & $1.06-2.03$ & $0.0253 *$ & $\begin{array}{l}\text { No: } 30 \\
\text { Yes: } 35\end{array}$ & 0.7167 & $0.38-1.30$ & 0.2817 \\
\hline
\end{tabular}

aZ-scores were calculated from STIM1 expression value in 154 COADs and 68 READs patients recruited by TCGA project. ${ }^{b}$ Odds ratio was calculated with using $\exp (\beta)$.

'95\% Confidence intervals.

"COAD and READ patients with Stage I, IIA and IIB were categorized as " 0 " group versus " 1 " group that included patients on Stage IIIA, IIIB, IIIC, IV and IVA.

"COAD and READ patients with T1, T2 were categorized as "0" group versus "1" group that included patients on T3, T4a and $\mathrm{T} 4 \mathrm{~b}$.

${ }^{\mathrm{f}}$ Multinomial distribution is fitted. N1/N1a/N1b vs N0 and N2/N2a vs N0.

${ }^{g}$ M0 vs M1/M1a. Significant $P$-value was in bold*.

fit the rounded RPKM values into a normal distribution. After normalization, a moderated $t$-test was applied to identify DEGs and an FDR-adjusted $p$ value threshold of 0.1 was applied for filtering. Surprisingly, the RNAsequencing profile identified 3482 upregulated DEGs in COADs but 0 in READs, and 517 downregulated DEGs in COADs and 0 in READs (Supplementary Table S1B), which is similar in character to the microarray profile. A corresponding hierarchical clustering analysis also disclosed a discernible pattern in COADs (Figure 3A) but not READs (Figure 3B).

The SPIA [9], based on the KEGG pathway database [10], was applied to gain biological insights into calcium signaling pathways in COADs and READs. In COADs, the calcium signaling pathway was significantly overrepresented (Figure 3C, Supplementary Table S5A, Supplementary Figure S4A), and the corresponding FDRadjusted $p$ value was equal to 0.0104 (Supplementary Table S6). Note that the aberration of the calcium signaling pathway in COADs was supported by over-representation evidence but not perturbation evidence. Comparatively, the calcium signaling pathway met neither the overrepresentation criterion nor the perturbation criterion
(Figure 3D, Supplementary Table S5B, Supplementary Figure S4B) in READs.

Furthermore, in COADs, STIM1 overexpression was most highly correlated with upregulation of ARHGAP1/Cdc42GAP (FDR-adjusted $p=8.55 \times 10^{-7}$, estimated beta $=14.64)$, a Rho GTPase-activating protein which participates in RhoGTPase signaling pathway that is implicated in the EMT, showed extensive function on regulating cell proliferation, migration, invasion, adhesion, and apoptosis [11]. Although the role of ARHGAP1 in $\mathrm{CRC}$ has remained obscure, alteration of ARHGAP1 might contribute to tumor aggression (Figure $3 \mathrm{E}$ ).

Concordant expressions between different platforms (microarray and RNA-sequencing) were analyzed. We intersected upregulated DEGs and downregulated DEGs across different platforms and found 87 consistently upregulated and 8 consistently downregulated DEGs (Figure 3F). In other words, these 95 DEGs were successfully validated across different expression platforms, and the corresponding genes are shown in Figure 3G.

These results indicated a significant enrichment of the calcium signaling pathway in COADs but not ROADs, further supporting the inference of CRC subtype-specific 
A
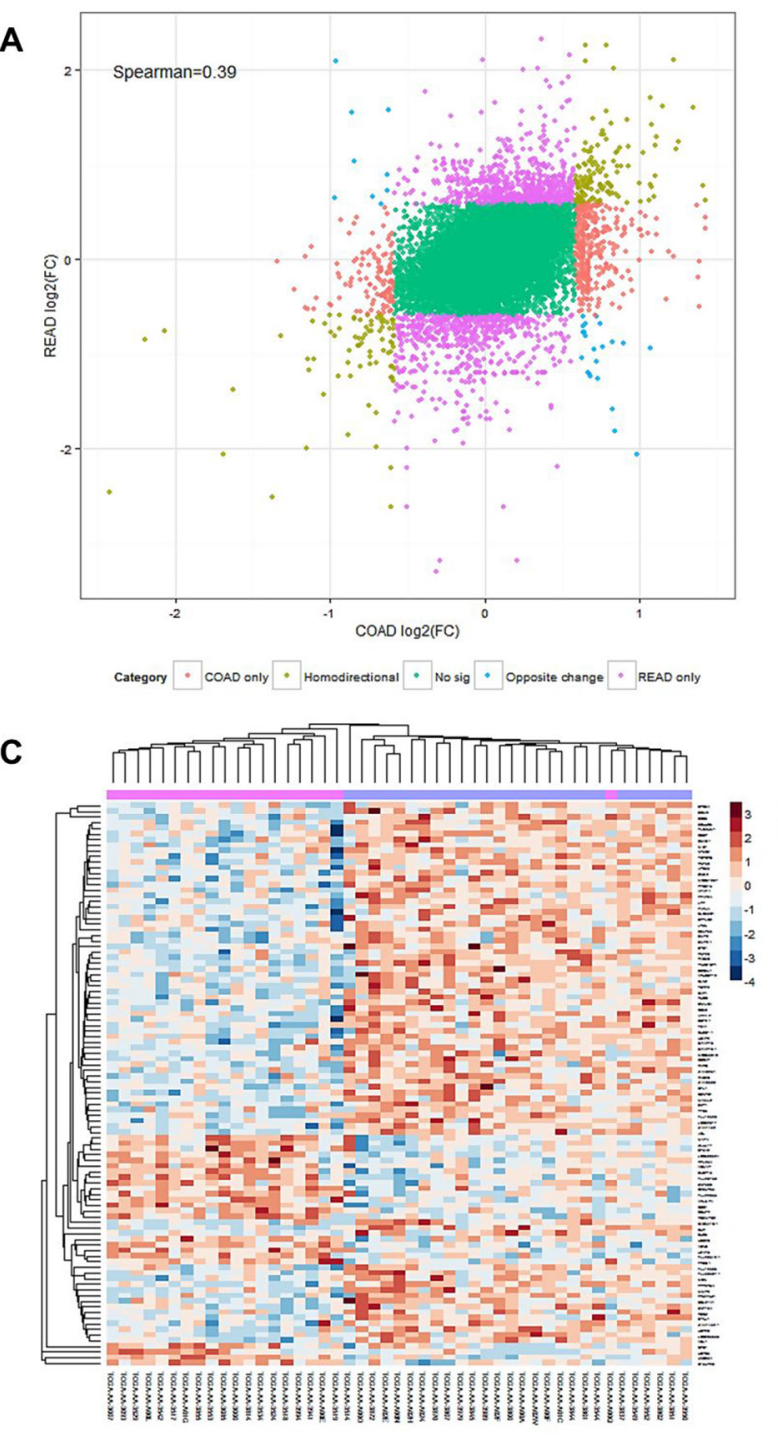

B

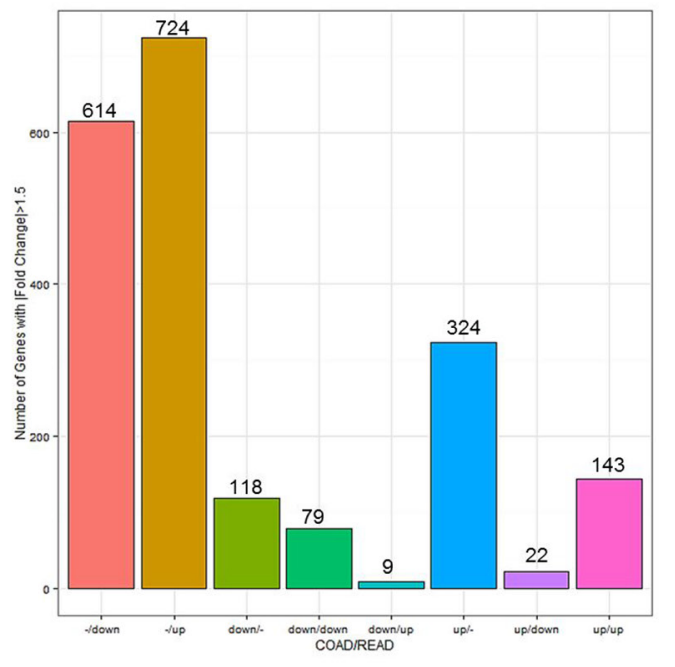

D

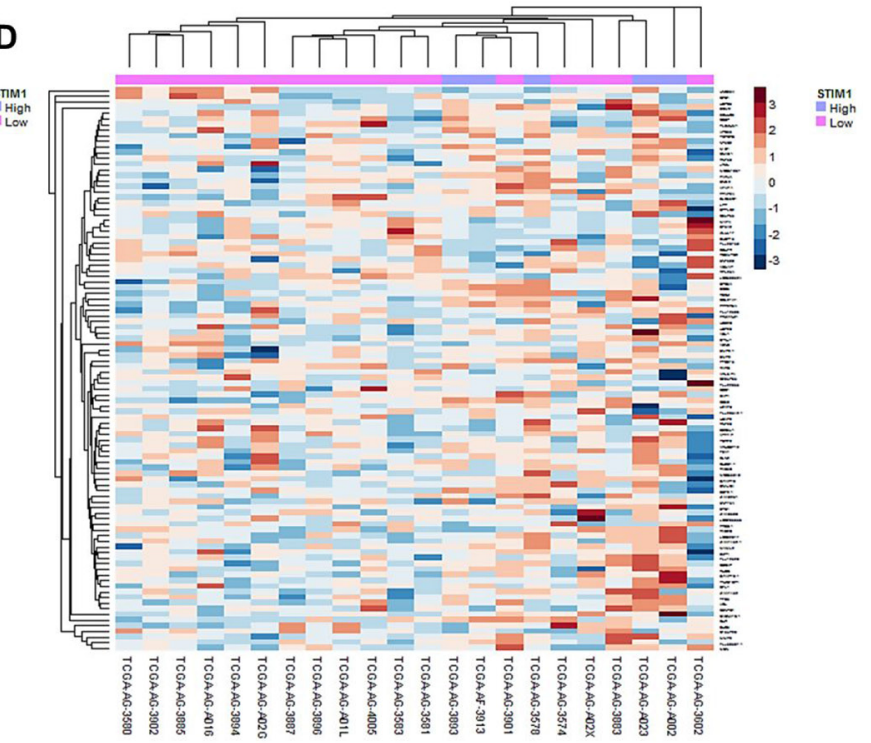

Figure 2: Expression landscape of colorectal cancer subtypes, colon adenocarcinomas (COADs) and rectal adenocarcinomas (READs). A. Starburst plot of each gene mapped according to its $\log 2$ multiple of change value in COADs (x-axis) and READs (y-axis). Genes with $\mid \log 2$ multiple of change| of $>1.5$ were selected to examine co-expression patterns. Red: genes up- or downregulated only in COADs. Purple: genes up- or-downregulated only in READs. Brown: genes changing in the same direction in both COADs and READs. Blue: genes changing in opposite directions in COADs and READs. The Spearman rank correlation of coefficient of expression value between COADs and READs was 0.39. B. Summarized histogram of selected genes in COADs and READs based on the criterion of a $\mid \log 2$ multiple of change| of $>1.5$. C. Heatmap of the top 100 most variant differentially expressed genes (DEGs) in COADs based on the microarray profile. Each row represents the top 100 most significant DEGs, and each column shows patient samples. A hierarchical clustering analysis was performed, and patient information based on STIM1 expression status was mapped. D. Heatmap of the top 100 most variant DEGs in READs based on the microarray profile. Each row represents the top 100 most significant DEGs, and each column shows patient samples. A hierarchical clustering analysis was performed, and patient information based on the STIMI expression status was mapped.

characteristics of STIMI-mediated SOCE pathway changes, and the relevance of STIMI-associated $\mathrm{Ca}^{2+}$ signaling to COADs but not READs.

\section{Association of STIM1 overexpression with miRNA in COAD and READ patients}

Besides mRNA expression levels, miRNA profiles of the CRC cohort were also studied. COAD and READ patients with available miRNA sequencing data were stratified into a STIMI overexpression group and STIMI low-expression group. In total, $80 \mathrm{COAD}$ patients (including 40 STIM1 overexpression and 40 STIM1 lowexpression) and 32 READ patients (including 11 STIM1 overexpression and 21 STIM1 low-expression) were selected.

The contrast based on the presence or absence of STIM1 overexpression was applied to calculate 

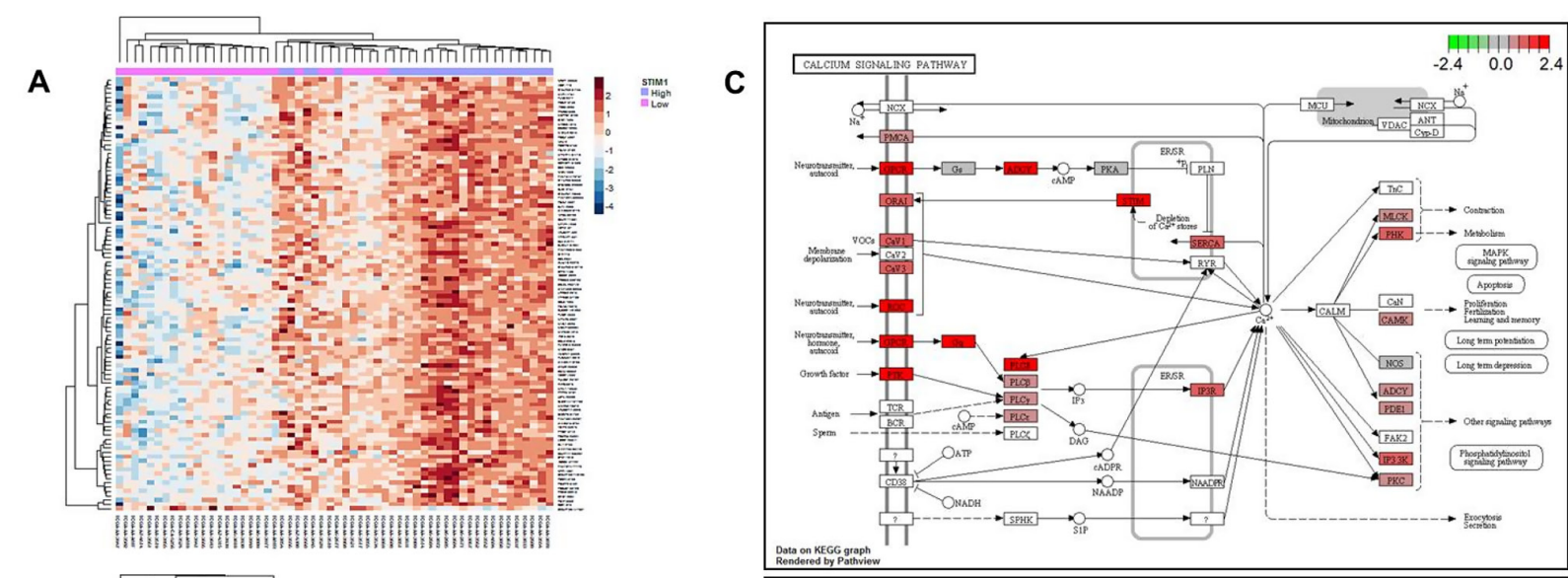

B

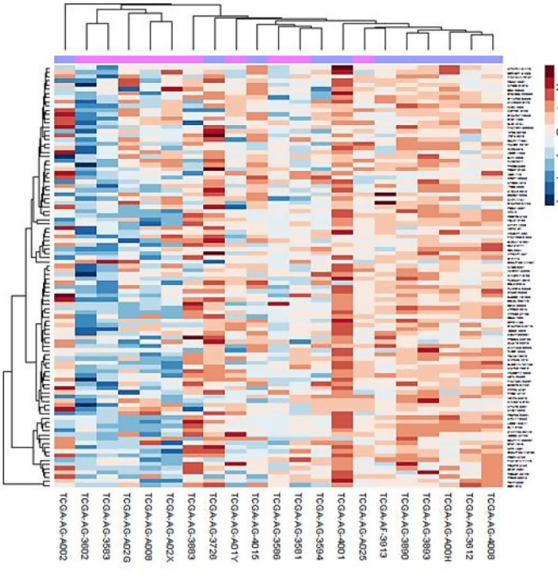

E

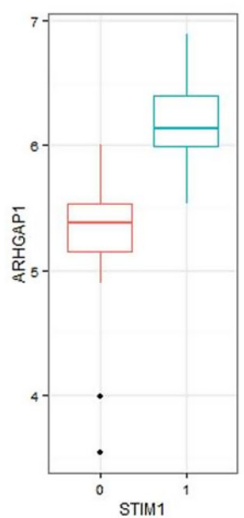

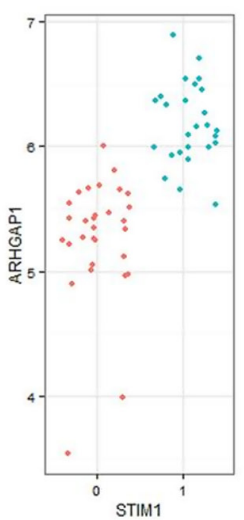

D

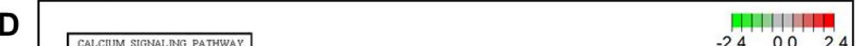

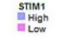

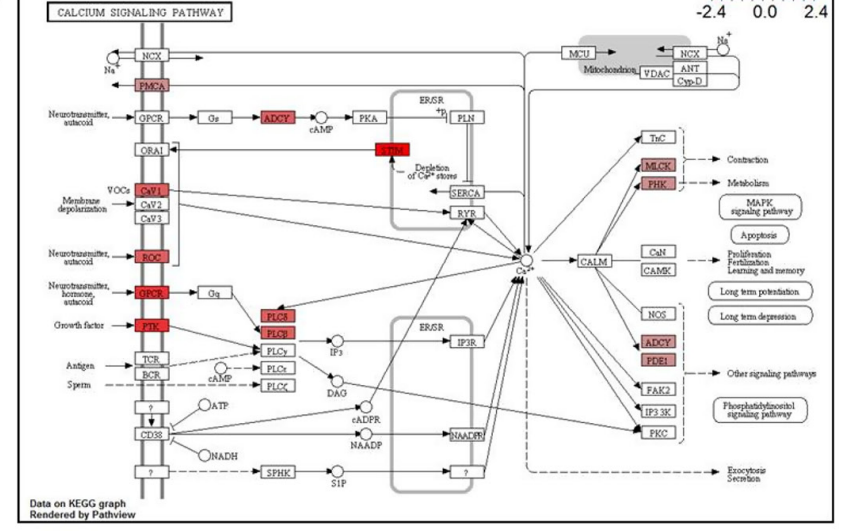

$\mathbf{F}$

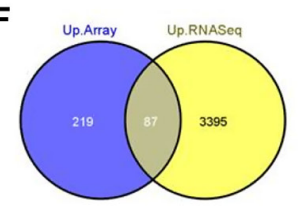

G

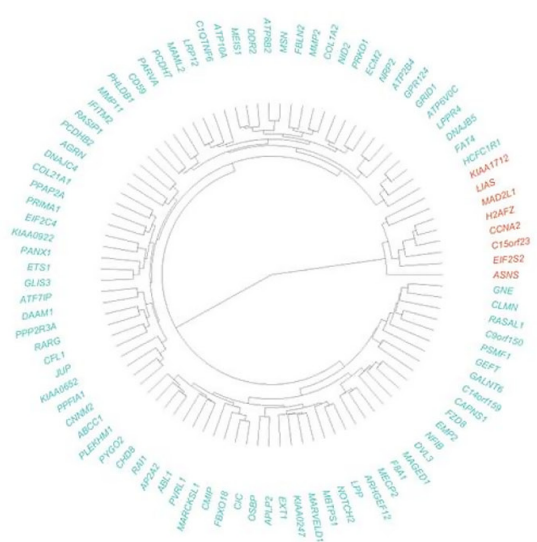

Figure 3: Validation of differential expression results by RNA-sequencing platform and a signaling pathway impact analysis (SPIA). A. Heatmap of the top 100 most variant differentially expressed genes (DEGs) in colon adenocarcinomas (COADs) based on the RNA sequencing profile. Each row represents the top 100 most significant DEGs, and each column shows patient samples. A hierarchical clustering analysis was performed, and patient information based on the STIM1 expression status was mapped. B. Heatmap of the top 100 most variant DEGs in rectal adenocarcinomas (READs) based on the RNA sequencing profile. Each row represents the top 100 most significant DEGs, and each column shows patient samples. A hierarchical clustering analysis was performed, and patient information based on the STIM1 expression status was mapped. C. Calcium signaling pathway of COADs based on the KEGG graph. The degrees of $\log 2$ multiples of change were mapped by red or green color based on the direction of the log2 multiple of change. D. Calcium signaling pathway of READs based on the KEGG graph. The degrees of $\log 2$ multiples of change were mapped by red or green color based on the direction of the $\log 2$ multiple of change. E. Whisker boxplot and jitter plot summarizing differences of normalized ARHGAPI expression levels in STIMI overexpression and STIMI low-expression groups (false discovery rate-adjusted $p=8.55 \times 10^{-7}$ ). In x-axis, 1 : STIM1 overexpression; 0: STIM1 under-expression. F. Venn diagrams showing the number of intersecting significantly differentially up- or downregulated genes across the microarray platform and RNA sequencing platform in COADs. Plots were constructed using Venny (http:// bioinfogp.cnb.csic.es/tools/venny/index.html). G. Dendrogram of 95 validated genes (green: 87 upregulated genes; red: 8 downregulated genes) constructed by a dissimilarity matrix of expression values based on Pearson's correlations. Genes were ordered by a hierarchical clustering based on the Euclidean distance and average linkage. 
multiples of change of miRNA, and a negative binomial distribution was fitted to account for the non-normality and dependency of the variance on the mean [12]. Having estimated the multiples of change and calculated corresponding $p$ values of miRNA in each CRC subtype, a FDR-adjusted $p$ value threshold of 0.1 was applied to identify differentially expressed miRNAs across COADs and READs. As a result, 10 upregulated and 16 downregulated miRNAs were identified in COAD patients (Figure 4A, Supplementary Table S7A); while only 2 downregulated miRNAs (hsa-miR-1978 and hsamiR-203) showed a significant correlation in READ patients (Supplementary Table S7B).

The following regularized log transformation and hierarchical clustering analysis were carried out to inspect comprehensive miRNA regulation of COADs (Figure 4B-4C) and READs (Figure 4D-4E). However, no distinctive pattern was observed in COADs and READs, indicating a modest regulatory role of STIMI overexpression in the overall miRNA profile. In spite of the fact of undetectable global interference of miRNA expression, STIM1 overexpression indeed affected a small number of miRNAs in COADs and READs (Supplementary Table S7C).

\section{Identification of prognostic mRNA/miRNA signatures in COADs for clinical outcomes across different clinical subclasses}

Having determined the 95 genes that were consistently detected by microarray and RNA-sequencing platforms and 16 miRNAs detected by miRNA sequencing in COADs, we conducted a stringent multistep clinical subclass-based survival analysis, which was similar to the strategy proposed by Volinia et al. [13], to identify COAD prognostic mRNA/miRNA signatures (Figure 5A). Thus, survival analyses were separately adjusted according to clinical profiles ( $\mathrm{T}$ stage, $\mathrm{N}$ stage, $\mathrm{M}$ stage, and vascularand lymphatic-invasion status). Furthermore, only mRNA or miRNA that showed $p$ values of $<0.05$ in at least two clinical subclasses were considered significant. Therefore, mRNA or miRNA identified to be significant indicated that these signatures were correlated with the patient survival rate after adjusting for several clinical subclasses.

Consequently, 10 transcripts, including PANX1, ETS1, EXT1, C1QTNF6, PCDH7, GLIS3, MARVELD1, PPP2R3A, KIAA0247, and LRP12, and 1 miRNA (hsa-miR-195, Supplementary Figure S5) successfully passed the multistep procedure and were related to clinical outcomes across COAD patients (Supplementary Table S8).

In addition, we constructed a PCA algorithm to transform these 11 prognostic signatures into a linear form (a so-called prognostic predictor, Supplementary Figure S6A). The model performance was assessed by the AUROC curve. The ROC curve was used to define the best threshold, and then patients were stratified into high-risk or low-risk groups based on this threshold. In COAD patients, the prognostic predictor value of each patient was calculated by the model based on the expression value, and the best threshold (prognostic predictor $=2.3$ ) was calculated by the ROC curve (with a sensitivity of $100.0 \%$ and a specificity of $81.0 \%$ ). In addition, the AUROC curve of $92.4 \%$ revealed good performance on survival prediction using the prognostic predictor (Figure 5B). Thus, the survival rates of COAD patients (consisting of STIM1 overexpression and STIM1 low-expression) categorized into two groups based on the prognostic predictor were compared. The Kaplan-Meier estimator revealed a significant difference in survival rates across the 2 groups (Cox-proportional hazard $p=0.0083$, Figure 5C). Moreover, we collected all available COAD patients without considering the STIMI expression value to carry out a prognostic predictor-based survival analysis, and results revealed a decline in the ROC performance (AUROC $=65.8 \%$, Supplementary Figure S6B). The corresponding Kaplan-Meier estimator also revealed a decrease in the ability to separate high-risk and low-risk groups, although the prognostic predictor still attained a significant effect in survival prediction (Cox-proportional hazard $p=0.022$, Supplementary Figure S6C). These results indicated that the STIM1-related mRNA/miRNA signature participated in the transcriptomic foundation for survival, but could only explain a portion of the overall survival in all COAD patients.

Finally, to elaborate the association between the prognostic predictor and STIM1 expression status, we compared prognostic predictor values between the STIMI overexpression and STIM1 low-expression groups. COAD patients who fell within the high-risk group (prognostic predictor $>2.3$ ) consisted of a large portion of the STIM1 overexpression group $(87.5 \%, 14$ of 16$)$, whereas the low-risk group consisted of a larger number of the STIM1 low-expression group $(61.7 \%, 29$ of 47) (approximate Pearson's Chi-squared $p=0.001$, Figure 5D). An in-depth analysis also revealed a strong difference in prognostic predictor value between STIM1 expression groups (exact Wilcoxon Mann-Whitney rank sum $p=1.39 \times 10^{-5}$, Figure 5E). Therefore, STIM1 overexpression and STIM1related molecular signatures were tightly correlated with COAD patient prognoses, and this phenomenon was enormously significant especially in the STIMI overexpression group versus the STIM1 low-expression group.

\section{DISCUSSION}

Our findings have several important implications for understanding STIM1 overexpression in CRC. First, we clearly determined distinct transcriptomic subtypes of CRC and documented the existence of a subtype-specific STIM1 role in CRC. An aberration of the SOCE pathway was 
A

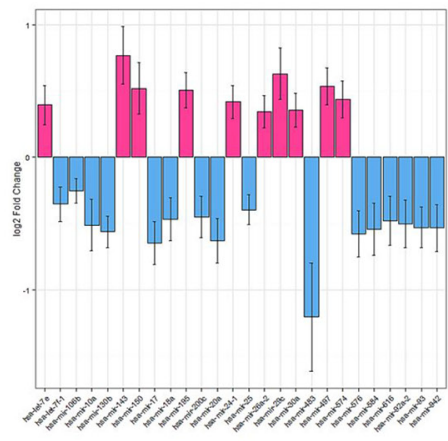

B

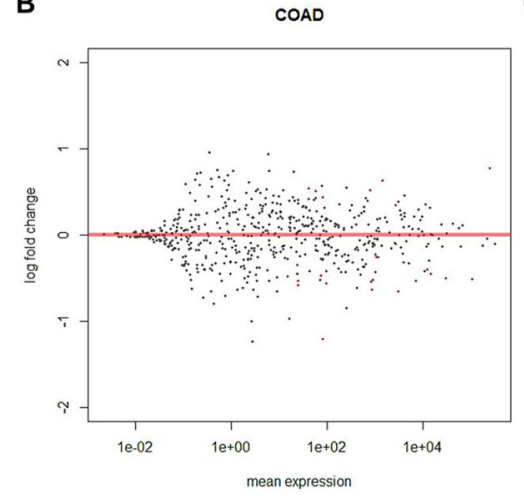

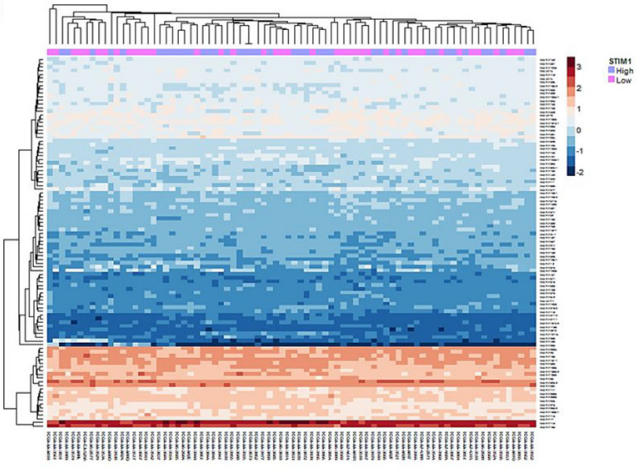

D

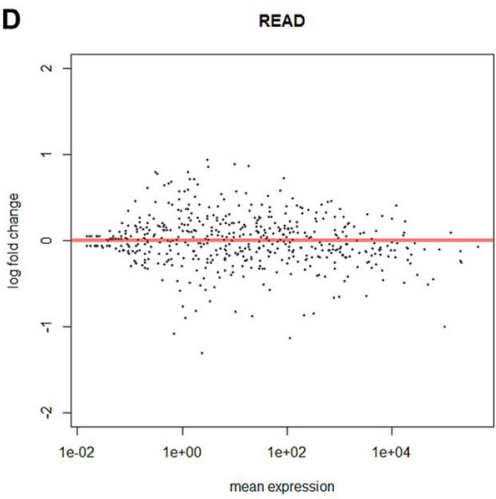

$E$

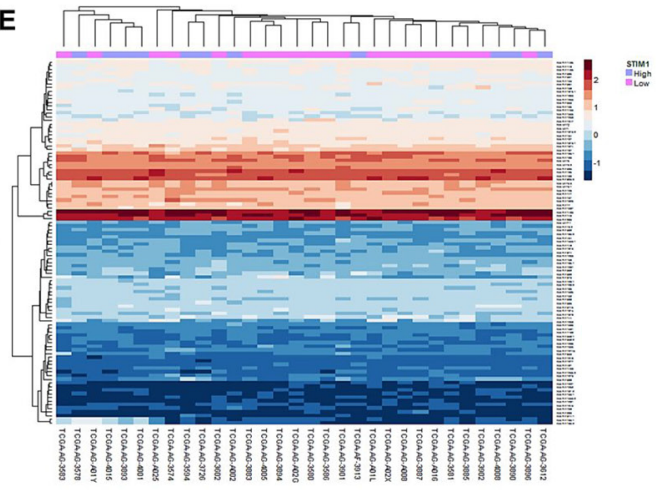

Figure 4: Analysis of colorectal cancer (CRC) patients' micro (mi)RNA expression profiles. A. Bar plot of significantly expressed miRNAs in colonic adenocarcinoma (COAD) patients and the corresponding $\log 2$ multiple of change value. The estimated standard error is represented by bars. B. MA plot of all miRNAs in COAD patients. The multiple of change was calculated based on the contrast between STIM1 overexpression and STIM1 low-expression, and miRNAs that passed the false discovery rate (FDR) threshold $($ FDR $<0.1)$ are in red color. C. Heatmap of the top 100 most variant miRNAs in COADs. Each row represents the top 100 most significant miRNAs, and each column shows patient samples. A hierarchical clustering analysis was performed, and patient information based on the STIM1 expression status was mapped. D. MA plot of all miRNAs in rectal adenocarcinoma (READ) patients. The multiple of change was calculated based on the contrast between STIM1 overexpression and STIM1 low-expression, and miRNAs that passed the FDR threshold $($ FDR $<0.1)$ are in red color. E. Heatmap of the top 100 most variant miRNAs in READs. Each row represents the top 100 most significant miRNAs, and each column shows patient samples. A hierarchical clustering analysis was performed, and patient information based on the STIM1 expression status was mapped.

previously described in CRC, and the STIM1-mediated $\mathrm{Ca}^{2+}$ oscillation was linked to tumor aggressiveness. However, the existence of CRC-subtype specific characteristics of the STIM1-mediated SOCE pathway remained elusive before this study. The combination of a dual-platform (microarray and RNA sequencing) genome-wide approach robustly discriminated STIMI-associated signatures as a feature of COADs but not READs in CRC patients. COAD patients have a profound increase in morbidity compared to READ patients, but the underlying global molecular mechanisms corresponding to this difference are unclear.

In this study, we observed a correlation between STIM1 expression values (z score) and lymphatic invasion in COADs. However, no significant correlation between STIM1 level and TNM and disease stage was identified. In compared with Wang et al. works [14], the STIM1 staining intensity was correlated with metastasis and disease stage. We noted that different STIM1 measurement approaches (mRNA transcript level by microarray versus protein staining) may contribute to the inconsistent findings.

In cancer cells, secondary messenger $\mathrm{Ca}^{2+}$ regulation of widespread physiological and pathological processes include tumor dissemination. The SOCE pathway was identified as the major $\mathrm{Ca}^{2+}$ entry mechanism in tumor cells. A decrease in the $\mathrm{ER} \mathrm{Ca}^{2+}$ concentration further triggers STIM1, a calcium sensor, to aggregate and translocate to cell membranes. Orai1, a plasma membrane store-operated calcium channel, is activated by STIM1 to allow $\mathrm{Ca}^{2+}$ influx. Consequently, STIM1-regulated $\mathrm{Ca}^{2+}$ influx facilitates focal adhesion turnover [5] and controls invadopodium formation and activity [4]. Significantly, STIMI regulates cancer cell migration and invasion ability, and this concept was supported by our data, which showed a correlation between elevated STIM1 expression levels and an enhanced lymphatic invasion status in COAD patients. Using computational tools, we also observed a significant SOCE pathway perturbation resulting from STIMI overexpression in COADs, which modulates the biological processes 
A

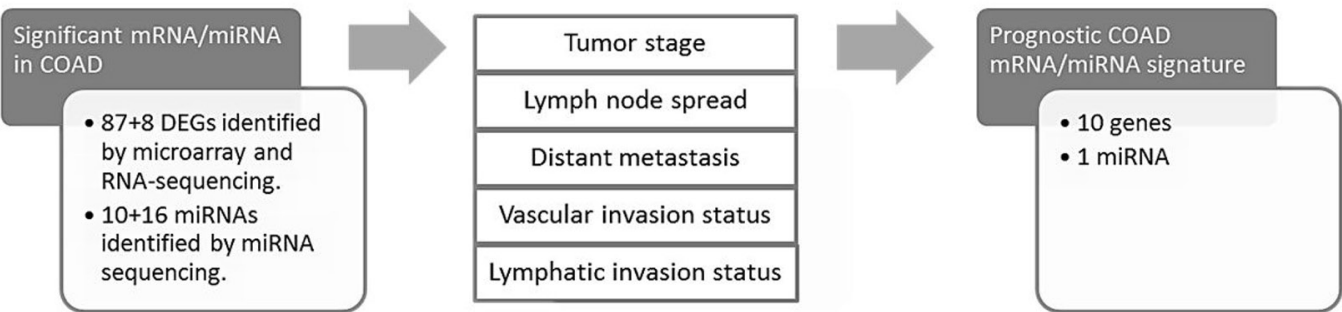

B

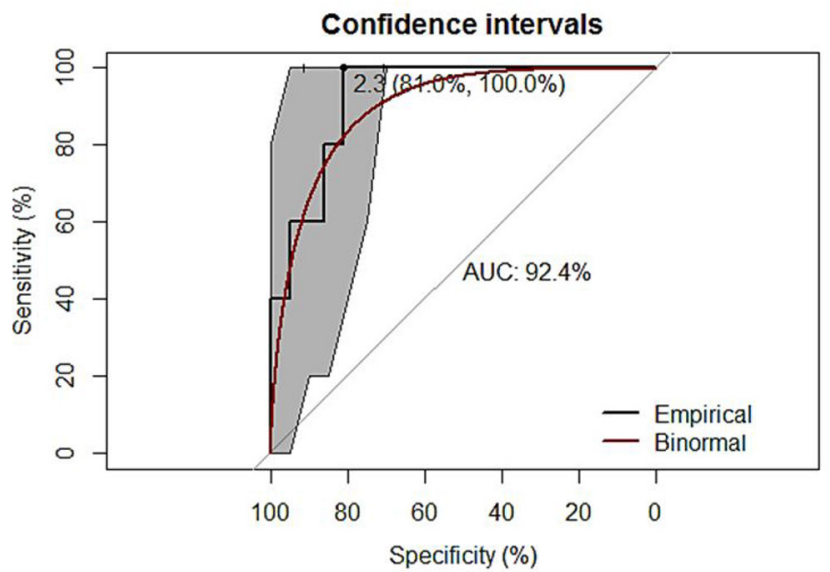

C

Kaplan-Meier of mRNA/miRNA risk predictor

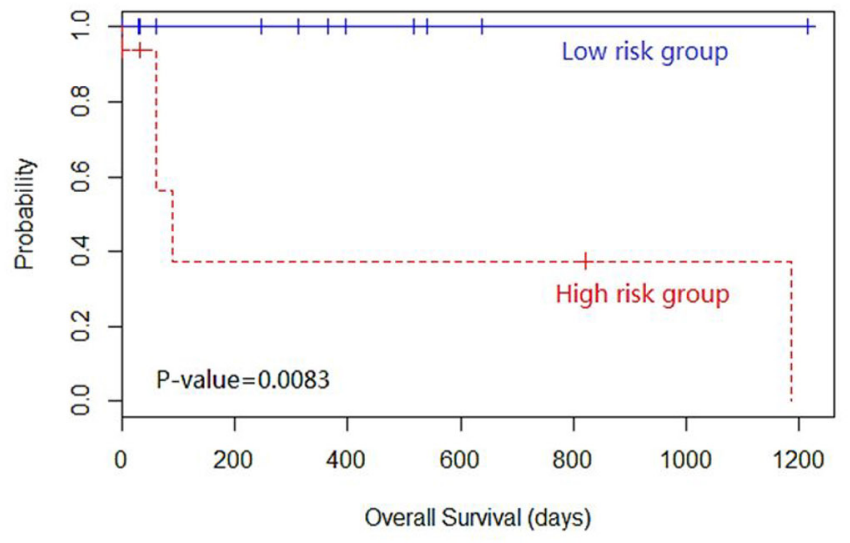

D

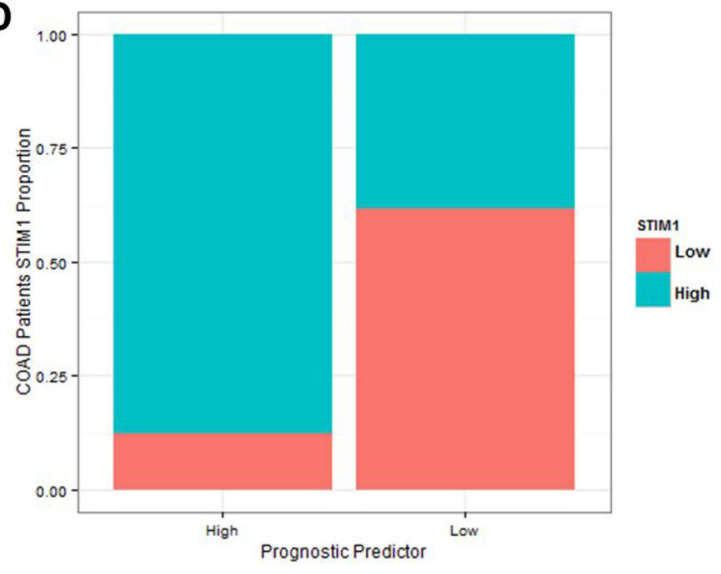

E

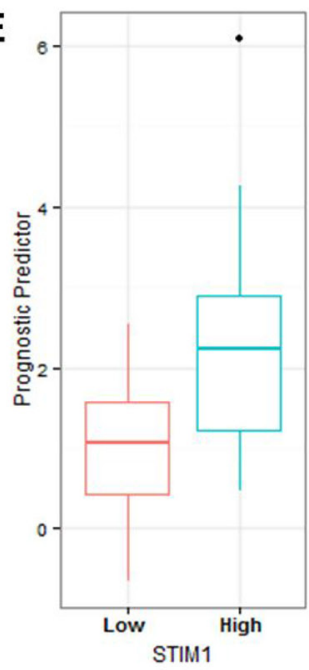

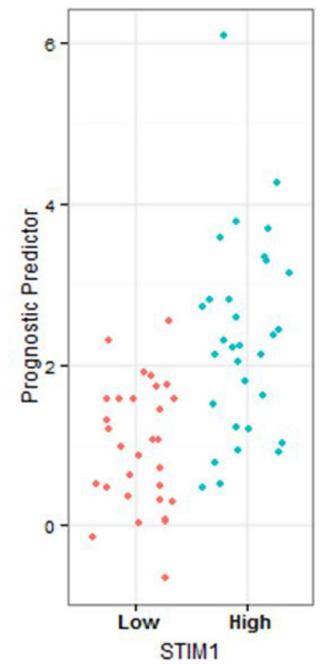

Figure 5: Analysis of the prognostic mRNA/micro (mi)RNA signature identification in 63 colon adenocarcinoma (COAD) patients and their performance in survival prediction. A. Scheme of the survival analysis strategy. A survival analysis was performed on the following clinical subclasses: tumor stage, lymph node pathologic spread, distant metastasis, vascular invasion, and lymphatic invasion. In total, 95 mRNAs and 26 miRNAs that were significant in at least 2 clinical subclasses were considered significantly associated with patient survival. B. Receiver operating characteristic (ROC) curve of prognostic predictors with 1-specificity as the $\mathrm{x}$-axis and sensitivity as the y-axis. The $95 \%$ confidence interval of the ROC curve was bootstrapped by DeLong's method to confirm the significance of the ROC curve. The best threshold (prognostic predictor $=2.3)$ and corresponding specificity and sensitivity $(81.0 \%$ and $100.0 \%$, respectively) are shown. In addition, the area under the ROC (AUROC) curve of $92.4 \%$ is shown. The binomial smoothed line is shown in red by the binomial method. C. Kaplan-Meier curve for overall survival for dichotomized prognostic predictor. $p$ values were calculated by the Cox regression analysis of STIM1 overexpression group and STIM1 low-expression group. D. Correlation between the presence or absence of STIM1 overexpression and the dichotomized prognostic predictor. E. Whisker boxplot and jitter plot for comparison between the presence or absence of STIM1 overexpression and the continuous prognostic predictor.

of cell migration and cell motility. Therefore, STIMI overexpression implicates a hyperactive SOCE pathway in COADs and demonstrates a higher propensity for invasion. However, comparing between STIM1 overexpression and
STIM1 low-expression groups of READ patients did not reveal dysregulation of the SOCE pathway, unraveling a minor role of STIMI and the SOCE pathway in ROAD tumor dissemination. In Li et al.'s study [3], the calcium 
signaling pathway was one of the downregulated pathways in READs, which was consistent with our SPIA results: a negative direction (inhibition) of the SOCE pathway perturbation. Comparing differences in STIM1 expression values in CRC subtypes indicated a slight increase in STIM1 overexpression patients in COADs, and a slight increase in STIM1 low-expression patients in READs. These results should be carefully interpreted, as the divergence of the STIMI overexpression status in CRC subtypes was small, and the difference in the degree of impact of the SOCE pathway in CRC subtypes might not be simply and completely attributed to discrepancies in STIMI distribution. A possible explanation of the dissimilarity expression profiles across COADs and READs could be that STIM1 overexpression might be due to a consequence of an aberration of different multiple upstream signaling pathways, and downstream influences of STIM1-mediated $\mathrm{Ca}^{2+}$ changes were multifaceted and multifactorial.

In the GSEA analysis, the ectopic STIM1 profile showed positive enrichment in immune system processes in COADs and a negative correlation was noted in READs. In pathophysiological aspects, STIM1-mediated $\mathrm{Ca}^{2+}$ signaling and the SOCE pathway play critical roles in regulating immune responses, and activation of the SOCE pathway through STIM1 overexpression dramatically worsens the proinflammatory status $[15,16]$. Increasing evidence indicates that STIMI-mediated cyclooxygenase (COX)-2 overexpression, an important inducible proinflammatory enzyme, might exacerbate tumor migration and progression [17]. In addition, impairment of regulation of the immune system is tightly bound to clinical outcomes of CRC. Therefore, our results suggest that STIM1-mediated $\mathrm{Ca}^{2+}$ signaling and STIMI overexpression might be prospective therapeutic targets for COAD treatment. In the network analysis, the module included genes correlated with lymphatic invasion that were also identified. Significantly, a large number of proteins in this network (MAPK9, MAP2K4, MAPK10, MAP4, MARK4, TGFBR2, and so on) were associated with mitogen-activated protein kinase (MAPK) pathways, which are well-known for their role in CRC. However, associations between STIMI and MAPK signaling pathways are ill-defined. Our data illustrated a possibility of interaction between the calcium signaling pathway and MAPK signaling pathway in COADs. Furthermore, Fan et al. showed the migration-promoting effect of a CRC cell line of SERCA overexpression via activation of the MAPK signaling pathway, further suggesting direct crosstalk between $\mathrm{Ca}^{2+}$ signaling and MAPK signaling [18]. In addition, alpha-actinin 1 (ACTN1), one of the genes identified in the network, is associated with focal adhesion formation, and its phosphorylation modulates pressureinduced adhesion in colon cancer cells [19]. Activating transcription factor 7 (ATF7IP), another transcription factor proven to be related to lymphatic invasion in our study, is involved in telomerase expression mediated by
Sp1 [20]. In short, we successfully identified an integrated network module which was mostly positively correlated with a dysregulated STIMI signature, which constituted the STIM1-associated invasiveness nature of COADs.

Beyond the mRNA data, COAD-associated miRNAs were discovered by a miRNA sequencing analysis. Specifically, loss of $h s a-m i R-10 a$ that targets $K L F 4$, as seen in STIM1 overexpression patients, led to upregulation of LPO and initiation of colorectal carcinomas [21]. hsa-miR-130b was reported to suppress CRC invasion and migration by downregulating integrin $\beta 1$ [22], and downregulation of $h s a-m i R-130 b$ was observed in the STIM1 overexpression group in COADs. hsa-miR-18a, that targets $\mathrm{CDC} 42$ and acts as a tumor suppressor, was significantly downregulated in STIMI-enriched patients [23]. hsa-miR-200c, which was downregulated in the STIM1 overexpression group, is associated with proliferation, migration, and invasion in CRC cell lines [24]. In addition, upregulation of ANGPTL2, which is associated with downregulation of $h s a-m i R-25$, was correlated with reductions in the invasive and migratory abilities of human CRC [25]. Furthermore, downregulation of hsa-miR-93 was observed, which promotes colon cancer development via upregulation of the Wnt/ $\beta$-catenin pathway [26]. In READs, hsa-miR-203 downregulation is associated with upregulation of Snail and improved invasion or metastasis potential of CRC cell lines [27].

In this study, we made our hypothesis based on cancer cells but not stroma cells in cancer tissues. The limitation of our study is that we could not clarify the STIM1 expression in cancer cells or that in stroma cells. However, STIM1 level is unlikely to be dominant in stroma cells because STIM1 was also highly expressed in cancer cells (data not shown).

The genomic basis of prognostic STIMI-associated signatures is unclear. Our results demonstrated that dysregulation in the STIMI-associated transcriptome explains a proportion of the differences between COADs and READs that underlie these signatures. The compact correlation of these changes with a STIM1-concerted expression phenotype plays a critical STIM1-centered role in predicting clinical outcomes. In addition, the transcriptome profiles we derived and the associated STIMI-associated signatures provide a previously unknown mechanistic link between CRC subtypes with differing invasive behaviors and transcriptional signatures that predict clinical prognostic outcomes. The data herein indicate a straightforward correlation between STIM1-mediated $\mathrm{Ca}^{2+}$ signaling and wide-ranging signatures known to be associated with tumor aggression, and this implication was only present in COADs. In summary, this study provides a comprehensive transcriptomic framework for understanding STIM1-related mRNA/miRNA signatures present in CRC subtypes with differing invasive behaviors, and suggests that $\mathrm{Ca}^{2+}$ signaling-targeted therapy may help further perfect the clinical capability to implement precision medicine for CRC patients. 


\section{MATERIALS AND METHODS}

\section{Clinical and integrated profiles of the TCGA CRC cohort and related patients}

Having determined patient categorization, we queried COAD and READ samples based on the STIM1 expression value (z-score) using the cBio Cancer Genomics Portal (http://www.cbioportal.org/). Sample data of different technical platforms (microarray, RNA-sequencing, and miRNA sequencing) were downloaded from the TCGA website (https://tcga-data.nci.nih.gov/tcga/) in data level 3 or a data matrix [7]. Following the procedures of Tell et al. [28], transcriptomic profiles were studied in patients of each CRC subtype, and patient categorization was carried out based on STIM1 z-scores. In this study, we defined CRC patients with a STIM1 z-score of $>+1$ as the overexpression group (STIM1+), and those with a STIM1 z-score of $<-1$ as the low-expression group (STIM1-). Simultaneously, comparisons between STIM1+ and STIM1- were performed across different experimental types.

\section{Analysis of clinical features of CRC patients}

Extended clinical demographics including cancer stage and survival data were acquired from the TCGA data portal. In total, $154 \mathrm{COAD}$ and 68 READ patients were included to assess the correlation between STIM1 expression values and clinical features. A logistic regression model under a quasibinomial distribution was fitted for the association test in COAD and READ patients. According to the disease stage, patients were categorized into 2 groups (stages I, IIA, and IIB vs. stages IIIA, IIIB, IIIC, IV, and IVA). Similarly, patients were categorized into a $\mathrm{T} 1$ and $\mathrm{T} 2$ group and a T3, T4a, and T4b group according to the $\mathrm{T}$ stage. In addition, a multinomial logistic regression was applied to identify correlations between STIM1 and the N stage (N0 vs. N1 vs. N2).

\section{Analysis of microarray and RNA-sequencing profiles of CRC patients}

Microarray data including $47 \mathrm{COAD}$ and $22 \mathrm{READ}$ samples and RNA-sequencing data including 56 COAD and 21 READ samples with available STIM1 expression value were subjected to data sanitization. Then, we imputed missing values by the kth-nearest neighbors (k-NN) algorithm. As the downloaded microarray data had already been normalized, we identified differentially expressed genes (DEGs) using a moderated $t$-test [29] by comparing STIM1+ and STIM1- patients who had COADs and READs. Significant DEGs were defined as genes having a false discovery rate (FDR)-adjusted $p$ value threshold of $<0.1$. Unsupervised hierarchical clustering was carried out on COADs and READs with the top 100 most variant DEGs identified in COADs. Note that the top 100 DEGs in READs were not analyzed because none of them passed the statistical cutoff point, and thus the analysis would have been nonsensical. We then applied the non-negative matrix factorization (NMF) method to validate the clustering effect [30] of the top 100 most variant DEGs using Brunet et al.'s algorithm in COADs [8]. In the NMF analysis, 200 runs were iteratively performed to identify the stability of the consensus matrix under different factorization ranks of 2 to 6 . For RNA-sequencing data, the value of reads per kilobase per million reads (RPKM) was provided. In order to fit the RPKM to the downstream DEG identification analysis, we normalized the RPKM value with a rounding cutoff of 0.1 and $\log 2$ transformation [31]. DEGs were identified by a moderated $t$-test with an FDR-adjusted $p$ value threshold of 0.1 .

\section{Gene set enrichment and interactome analysis of DEGs in CRC patients}

Following expression analysis, a gene ontology (GO) biological process (BP) term analysis was performed [32]. We separately applied the gene set enrichment and analysis (GSEA) algorithm [33] to identify enriched $\mathrm{BP}$ terms in COADs and READs. After permutation, BP terms were filtered by the following criteria in both CRC subtypes: an FDR value of $<5 \times 10^{-5}$, a gene set size of $>150$, and an absolute value of subtracted normalized enrichment score (NES) of $>1.2$. Remaining BP terms were then compared between COADs and READs. In interactome aspects, a network analysis was conducted to identify differentially expressed network modules corresponding to the lymphatic invasion phenotype (see Supplementary Note) [34]. We used Human Protein Reference Database (HPRD) to provide protein-protein interaction information [35]. We then aggregated $p$ values and fit a beta-uniform mixture (BUM) distribution. Scored nodes in the network were then used to find a maximum scoring subnetwork with a heuristic algorithm [36].

\section{Signaling pathway impact analysis (SPIA) of DEGs in CRC patients}

We carried out an signaling pathway impact analysis (SPIA) to analyze the difference of aberrant pathways between COADs and READs using RNA sequencing data [9]. In COADs, 3648 DEGs based on an FDR threshold of 0.1 were selected for the over-representation analysis (ORA), a part of the analytic workflow implemented in SPIA; however, 1215 genes with $p$ values of $<0.05$ in READs were subjected to an ORA analysis as no gene passed FDR filtering. In addition, the number of bootstrap iterations used to compute the perturbation $p$ value was 20,000 , and Fisher's combined method was used to combine the over-representation $p$ values and perturbation $p$ values [37]. 


\section{Analysis of miRNA sequencing profile in CRC patients}

We tested for associations of miRNA-STIMI expression levels by assuming a negative binomial distribution using a generalized linear model [12]. In total, $80 \mathrm{COAD}$ and $32 \mathrm{READ}$ patients were included in the miRNA analysis. The raw miRNA read counts were directly applied for differentially expressed miRNA identification. We set the significance threshold of FDR-adjusted $p$ values to 0.1 . We then regularized log transformation of count data to undergo downstream heatmap and hierarchical clustering analyses.

\section{Survival analysis of significant $\mathrm{mRNA} / \mathrm{miRNA}$ signatures}

In COADs, the correlation between RNA expression and overall survival was conducted by a modified strategy which was similar to that proposed by Volinia and Croce [13]. To avoid bias caused by an imputed expression level, we removed patients with at least one missing value of mRNA/miRNA signatures. Hazard ratios were calculated by Cox-proportional hazard coefficients. Clinical covariates were incorporated into the multivariate Cox-proportional hazard model to identify independent molecular RNA signatures. Therefore, the association results were reported as five clinical subclasses: tumor stage, lymph node spread status, distant metastasis, vascular invasion status, and lymphatic invasion status. To evaluate the prognostic value of the identified mRNA/miRNA signatures, we used a principal component analysis (PCA) algorithm to calculate the linear combination of corresponding molecular signatures. The best threshold and performance of prognostic predictor were determined by a receiver operating characteristic (ROC) curve and area under the ROC (AUROC) curve, respectively [38].

\section{Statistical analysis and annotation}

We used R (http://www.r-project.org/; http://cran.rproject.org/) and Bioconductor (http://www.bioconductor. org/) for all analytic workflows in this study.

\section{ACKNOWLEDGMENTS AND FUNDING}

This work was supported by funding from an Excellence for Cancer Research Center grant, Department of Health, Executive Yuan, Taiwan, ROC (DOH102TD-C-111-002) and grants from the National Science Council, Taiwan, ROC (MOST 104-2320-B-038-016) and from Taipei Medical University (12310-0223).

\section{CONFLICTS OF INTEREST}

The authors declare they have no known conflicts of interest in this work.

\section{Author contributions}

H.W.S.C. and W.C.C. designed the study. H.W.S.C. performed bioinformatics. H.W.S.C. and W.C.C. analyzed and interpreted the data. H.W.S.C. and W.C.C. wrote the paper.

W.C.C contributed materials, analysis tools and functional data.

\section{REFERENCES}

1. Siegel, R., J. Ma, Z. Zou, A. Jemal. Cancer statistics, 2014. CA Cancer J Clin. 2014; 64:9-29.

2. Hanahan, D., R.A. Weinberg. Hallmarks of cancer: the next generation. Cell. 2011; 144:646-74.

3. Li, J.N., L. Zhao, J. Wu, B. Wu, H. Yang, H.H. Zhang, J.M. Qian. Differences in gene expression profiles and carcinogenesis pathways between colon and rectal cancer. J Dig Dis. 2012; 13:24-32.

4. Sun, J., F. Lu, H. He, J. Shen, J. Messina, R. Mathew, D. Wang, A.A. Sarnaik, W.C. Chang, M. Kim, H. Cheng, S. Yang. STIM1- and Orai1-mediated $\mathrm{Ca}(2+)$ oscillation orchestrates invadopodium formation and melanoma invasion. J Cell Biol. 2014; 207:535-48.

5. Yang, S., J.J. Zhang, X.Y. Huang. Orai1 and STIM1 are critical for breast tumor cell migration and metastasis. Cancer Cell. 2009; 15:124-34.

6. Wang JY, Chen BK, Wang YS, Tsai YT, Chen WC, Chang WC, Hou MF, Wu YC, Chang WC. Involvement of store-operated calcium signaling in EGF-mediated COX-2 gene activation in cancer cells. Cell Signal. 2012; 24:162-9.

7. Cancer Genome Atlas, N . Comprehensive molecular characterization of human colon and rectal cancer. Nature. 2012; 487:330-7.

8. Brunet, J.P., P. Tamayo, T.R. Golub, J.P. Mesirov. Metagenes and molecular pattern discovery using matrix factorization. Proc Natl Acad Sci U S A. 2004; 101:4164-9.

9. Tarca, A.L., S. Draghici, P. Khatri, S.S. Hassan, P. Mittal, J.S. Kim, C.J. Kim, J.P. Kusanovic, R. Romero. A novel signaling pathway impact analysis. Bioinformatics. 2009; 25:75-82.

10. Kanehisa, M., S. Goto. KEGG: kyoto encyclopedia of genes and genomes. Nucleic Acids Res. 2000; 28:27-30.

11. Zohrabian, V.M., H. Nandu, N. Gulati, G. Khitrov, C. Zhao, A. Mohan, J. Demattia, A. Braun, K. Das, R. Murali, M. Jhanwar-Uniyal. Gene expression profiling of metastatic brain cancer. Oncol Rep. 2007; 18:321-8.

12. Love, M.I., W. Huber, S. Anders. Moderated estimation of fold change and dispersion for RNA-seq data with DESeq2. Genome Biol. 2014; 15:550.

13. Volinia, S., C.M. Croce. Prognostic microRNA/mRNA signature from the integrated analysis of patients with invasive breast cancer. Proc Natl Acad Sci U S A. 2013; 110:7413-7. 
14. Wang, J.Y., J. Sun, M.Y. Huang, Y.S. Wang, M.F. Hou, Y. Sun, H. He, N. Krishna, S.J. Chiu, S. Lin, S. Yang, W.C. Chang. STIM1 overexpression promotes colorectal cancer progression, cell motility and COX-2 expression. Oncogene. 2015; 34:4358-67.

15. Samakai, E., R. Hooper, J. Soboloff. The critical role of STIM1-dependent Ca2+ signalling during T-cell development and activation. Int J Biochem Cell Biol. 2013; 45:2491-5.

16. Shaw, P.J., S. Feske. Physiological and pathophysiological functions of SOCE in the immune system. Front Biosci (Elite Ed). 2012; 4:2253-68.

17. Wang, D., R.N. DuBois. The role of anti-inflammatory drugs in colorectal cancer. Annu Rev Med. 2013; 64:131-44.

18. Fan, L., A. Li, W. Li, P. Cai, B. Yang, M. Zhang, Y. Gu, Y. Shu, Y. Sun, Y. Shen, X. Wu, G. Hu, X. Wu, et al. Novel role of Sarco/endoplasmic reticulum calcium ATPase 2 in development of colorectal cancer and its regulation by F36, a curcumin analog. Biomed Pharmacother. 2014; 68:1141-8.

19. Craig, D.H., B. Haimovich, M.D. Basson. Alpha-actinin-1 phosphorylation modulates pressureinduced colon cancer cell adhesion through regulation of focal adhesion kinase-Src interaction. Am J Physiol Cell Physiol. 2007; 293:C1862-74.

20. Liu, L., K. Ishihara, T. Ichimura, N. Fujita, S. Hino, S. Tomita, S. Watanabe, N. Saitoh, T. Ito, M. Nakao. MCAF1/AM is involved in Sp1-mediated maintenance of cancer-associated telomerase activity. J Biol Chem. 2009; 284:5165-74.

21. Stadthagen, G., D. Tehler, N.M. Hoyland-Kroghsbo, J. Wen, A. Krogh, K.T. Jensen, E. Santoni-Rugiu, L.H. Engelholm, A.H. Lund. Loss of miR-10a activates lpo and collaborates with activated Wnt signaling in inducing intestinal neoplasia in female mice. PLoS Genet. 2013; 9:e1003913.

22. Zhao, Y., G. Miao, Y. Li, T. Isaji, J. Gu, J. Li, R. Qi. MicroRNA- 130b suppresses migration and invasion of colorectal cancer cells through downregulation of integrin beta1 [corrected]. PLoS One. 2014; 9:e87938.

23. Humphreys, K.J., R.A. McKinnon, M.Z. Michael. miR$18 \mathrm{a}$ inhibits CDC42 and plays a tumour suppressor role in colorectal cancer cells. PLoS One. 2014; 9:e112288.

24. Lu, Y.X., L. Yuan, X.L. Xue, M. Zhou, Y. Liu, C. Zhang, J.P. Li, L. Zheng, M. Hong, X.N. Li. Regulation of colorectal carcinoma stemness, growth, and metastasis by an miR200c-Sox2-negative feedback loop mechanism. Clin Cancer Res. 2014; 20:2631-42.

25. Zhou, J., J. Wang, S. Wu, S. Zhu, S. Wang, H. Zhou, X. Tian, N. Tang, S. Nie. Angiopoietin-like protein 2 negatively regulated by microRNA-25 contributes to the malignant progression of colorectal cancer. Int J Mol Med. 2014; 34:1286-92.
26. Tang, Q., Z. Zou, C. Zou, Q. Zhang, R. Huang, X. Guan, Q. Li, Z. Han, D. Wang, H. Wei, X. Gao, X. Wang. MicroRNA-93 suppress colorectal cancer development via Wnt/beta-catenin pathway downregulating. Tumour Biol. 2015; 36:1701-10

27. Ju, S.Y., S.H. Chiou, Y. Su. Maintenance of the stemness in CD44(+) HCT-15 and HCT-116 human colon cancer cells requires miR-203 suppression. Stem Cell Res. 2014; 12:86-100.

28. Tell, R.W., C.M. Horvath. Bioinformatic analysis reveals a pattern of STAT3-associated gene expression specific to basal-like breast cancers in human tumors. Proc Natl Acad Sci U S A. 2014; 111:12787-92.

29. Wettenhall, J.M., G.K. Smyth. limmaGUI: a graphical user interface for linear modeling of microarray data. Bioinformatics. 2004; 20:3705-6.

30. Gaujoux, R., C. Seoighe. A flexible R package for nonnegative matrix factorization. BMC Bioinformatics. 2010; $11: 367$.

31. Warden, C.D., N. Kanaya, S. Chen, Y.C. Yuan. BD-Func: a streamlined algorithm for predicting activation and inhibition of pathways. PeerJ. 2013; 1:e159.

32. Yu, G., L.G. Wang, Y. Han, Q.Y. He. clusterProfiler: an $\mathrm{R}$ package for comparing biological themes among gene clusters. OMICS. 2012; 16:284-7.

33. Subramanian, A., P. Tamayo, V.K. Mootha, S. Mukherjee, B.L. Ebert, M.A. Gillette, A. Paulovich, S.L. Pomeroy, T.R. Golub, E.S. Lander, J.P. Mesirov. Gene set enrichment analysis: a knowledge-based approach for interpreting genome-wide expression profiles. Proc Natl Acad Sci U S A. 2005; 102:15545-50.

34. Beisser, D., G.W. Klau, T. Dandekar, T. Muller, M.T. Dittrich. BioNet: an R-Package for the functional analysis of biological networks. Bioinformatics. 2010; 26:1129-30.

35. Keshava Prasad, T.S., R. Goel, K. Kandasamy, S. Keerthikumar, S. Kumar, S. Mathivanan, D. Telikicherla, R. Raju, B. Shafreen, A. Venugopal, L. Balakrishnan, A. Marimuthu, S. Banerjee, et al. Human Protein Reference Database - update. Nucleic Acids Res. 2009; 37:D767-72.

36. Castro, M.A., X. Wang, M.N. Fletcher, K.B. Meyer, F. Markowetz. RedeR: R/Bioconductor package for representing modular structures, nested networks and multiple levels of hierarchical associations. Genome Biol. 2012; 13:R29.

37. Luo, W., C. Brouwer. Pathview: an R/Bioconductor package for pathway-based data integration and visualization. Bioinformatics. 2013; 29:1830-1.

38. Robin, X., N. Turck, A. Hainard, N. Tiberti, F. Lisacek, J.C. Sanchez, M. Muller. pROC: an open-source package for $\mathrm{R}$ and $\mathrm{S}+$ to analyze and compare ROC curves. BMC Bioinformatics. 2011; 12:77. 